MITSUBISHI ELECTRIC RESEARCH LABORATORIES

http://www.merl.com

\title{
Statistical Analysis of the UWB Channel in an Industrial Environment
}

\author{
Johan Karedal, Shurjeel, Wyne, Peter Almers, Fredrik Tufvession, Andreas F. Molisch
}

TR2005-074 September 2004

\begin{abstract}
In this paper we present a statistical model for the ultra-wideband (UWB) channel in an industrial environment. Based on a set of measurements in a factory hall, we find that the abundance of metallic scatterers causes dense multipath scattering. This can be seen to produce mostly Rayleigh distributed small-scale fading signal, with only a few paths exhibiting Nakagami distributions. For the power delay profile, we suggest a generalization of the Saleh-Valenzuela model where clusters with difference excess delays have different ray power decay constants; the decay constants follow a linear dependence on the delay. This model provides an excellent fit to the measured data. We also note that for non-line-of-sight scenarios at larger distances, several hundred multipath components need to be collected to capture $50 \%$ of the available energy.
\end{abstract}

IEEE Vehicular Technology Conference (VTC)

This work may not be copied or reproduced in whole or in part for any commercial purpose. Permission to copy in whole or in part without payment of fee is granted for nonprofit educational and research purposes provided that all such whole or partial copies include the following: a notice that such copying is by permission of Mitsubishi Electric Research Laboratories, Inc.; an acknowledgment of the authors and individual contributions to the work; and all applicable portions of the copyright notice. Copying, reproduction, or republishing for any other purpose shall require a license with payment of fee to Mitsubishi Electric Research Laboratories, Inc. All rights reserved.

Copyright (C) Mitsubishi Electric Research Laboratories, Inc., 2004

201 Broadway, Cambridge, Massachusetts 02139 



\title{
Statistical Analysis of the UWB Channel in an Industrial Environment
}

\author{
Johan Karedal $^{1}$, Shurjeel Wyne ${ }^{1}$, Peter Almers ${ }^{1,2}$, Fredrik Tufvesson ${ }^{1}$, and Andreas F. Molisch ${ }^{1,3}$ \\ ${ }^{1}$ Department of Electroscience, Lund University, Box 118, SE-221 00 Lund, Sweden, \\ 2 TeliaSonera AB, Box 94, SE-201 20 Malmö, Sweden, \\ ${ }^{3}$ Mitsubishi Electric Research Labs, 201 Broadway, Cambridge, MA 02139, USA. \\ Email: \{Johan.Karedal, Shurjeel.Wyne, Peter.Almers, Fredrik.Tufvesson, Andreas.Molisch\}@es.lth.se
}

\begin{abstract}
In this paper we present a statistical model for the ultra-wideband (UWB) channel in an industrial environment. Based on a set of measurements in a factory hall, we find that the abundance of metallic scatterers causes dense multipath scattering. This can be seen to produce mostly Rayleigh distributed small-scale fading signal, with only a few paths exhibiting Nakagami distributions. For the power delay profile, we suggest a generalization of the Saleh-Valenzuela model where clusters with different excess delays have different ray power decay constants; the decay constants follow a linear dependence on the delay. This model provides an excellent fit to the measured data. We also note that for non-line-of-sight scenarios at larger distances, several hundred multipath components need to be collected to capture $50 \%$ of the available energy.
\end{abstract}

\section{INTRODUCTION}

Ultra-wideband (UWB) spread spectrum techniques have attracted increasing interest in recent years [1], [2], [3]. UWB systems are often defined to have a relative bandwidth that is larger than $20 \%$ and/or an absolute bandwidth of more then 500 $\mathrm{MHz}$ [4]. Several qualities make them suitable for a variety of applications; the low spectral density that the spreading of the information over such a large frequency band implies decreases interference to other co-existing systems and makes interception of communication difficult. The former is important in commercial applications whereas the latter is of high interest for military applications. Another benefit of spreading the signal over a wide frequency range is that communications are made more reliable in environments that cause frequency-selective fading [5].

An important application for UWB systems is machine-tomachine communications in industrial environments, e.g., process control systems or supervision of storage halls. For this reason, the IEEE 802.15.4a group is currently developing a standard for these systems. In the development of this (as of any wireless) system, realistic channel models are an absolute necessity. As previous UWB measurement campaigns have been restricted to office and residential environments, also channel models exist only for those environments, see e.g., [6], [7]. Especially, the standardized IEEE 802.15.3a model [8] is valid only for office and residential environments. On the other hand, available narrowband channel models in industrial environments (e.g., [9]) cannot be used, because the behaviour of the narrowband and the UWB channel is remarkably different [10].

Due to the reasons mentioned above, there is an urgent need for a channel model for an UWB channel in industrial environments, covering the FCC-approved frequency band $3.1-10.6$ $\mathrm{GHz}$ [4]. In [11] we present example measurement results of our measurement campaigns, and point out important physical propagation processes. In this paper, we give a statistical model for the measured data suitable as a basis for system simulations. The outcome will also be used as input to the channel modelling group of IEEE 802.15.4a.

The rest of the paper is organized as follows: Section II describes the measurement technique and the measured environment whereas Section III concerns the processing of the measured data. Next, in Section IV, the statistical model based on our generalized Saleh-Valenzuela model is presented, and finally the paper is ended by a summary and conclusions in Section V.

\section{Measurement Setup And EnVironment}

The measurements performed in the frequency domain determined the complex channel transfer function $H(f)$ using a vector network analyser (HP 8720C). We covered a frequency range of 3.1 to $10.6 \mathrm{GHz}$ implying a delay resoultion of approximately 0.13 ns. Since the data were collected during two campaigns, where the first one used a frequency division of 1251 points (6 $\mathrm{MHz}$ between the samples) and the second one used 1601 points $\left(4.7 \mathrm{GHz}\right.$ between the samples), ${ }^{1}$ the results have different maximum resolvable delays, $167 \mathrm{~ns}$ (corresponding to $50 \mathrm{~m}$ path delay) and $213 \mathrm{~ns}$ (64 $\mathrm{m}$ path delay), respectively. The frequency range implies that, after data processing, each obtained impulse response can be divided into 1251 or 1601 delay bins of $0.13 \mathrm{~ns}$ each.

An omnidirectional conical monopole antenna was used as receiver and transmitter, respectively. In order to create a virtual uniform linear antenna array (ULA) with 7 elements at each end, each (single) antenna was moved in 7 steps along a rail using a stepper motor. The antenna element positions were separated by a distance of $50 \mathrm{~mm}$ (corresponding to half a wavelength at 3.1 $\mathrm{GHz}$ ) in the first campaign, and by $37 \mathrm{~mm}$ (half a wavelength at $4 \mathrm{GHz}$ ) in the second. Antennas and rails were mounted on 1 $\mathrm{m}$ high tripods, and placed at various positions in the measured environment.

Our measurements were performed in a small incinerator hall (see Fig. 1) of DSM Resins Scandinavia, a chemical company in Landskrona, Sweden. The hall has a floor area of $13.6 \times 9.1 \mathrm{~m}$ and a height of $8.2 \mathrm{~m}$, hence, the largest dimension is roughly one fourth of the maximum resolvable path delay. The hall can

\footnotetext{
${ }^{1}$ The reason for this was an attempt to avoid some aliasing effects that could be seen in the results of the first campign.
} 


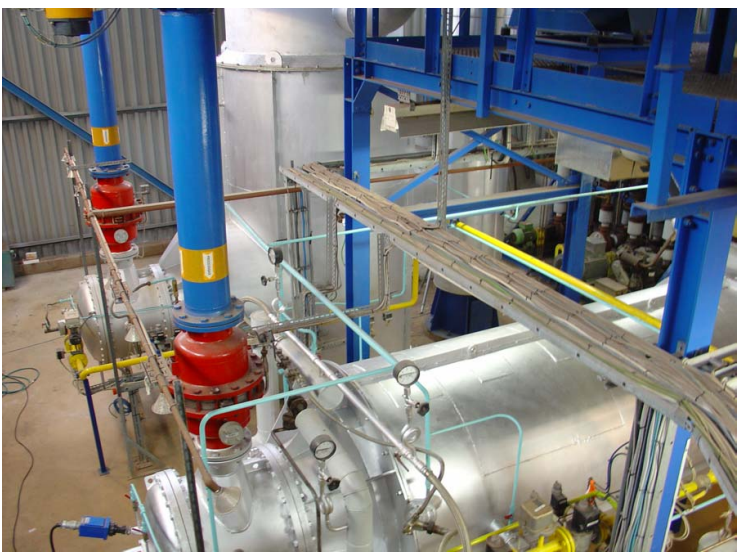

Fig. 1. A view of the incinerator hall, showing the metallic environment as seen from the balcony.

be said to represent a typical industrial environment in the sense that it is packed with metallic equipment, e.g., pumps, pipes and cannisters. Also, the walls and the ceiling consist of metal, in this case corrugated iron. Inside the hall, positions were selected to create three different scenarios, line-of-sight (LOS), non-lineof-sight (NLOS), and base station (BS) NLOS. In the latter, the transmitter tripod was placed on top of a $3 \mathrm{~m}$ high balcony located in one end of the building, whereas otherwise the transmitter and receiver were located at the same height constituting a peer-topeer scenario. In addition to the different scenarios, three different transmitter-receiver separations were chosen $(2 \mathrm{~m}, 4 \mathrm{~m}$, and $8 \mathrm{~m})$. In all, ten different measurements were performed.

Due to the crowded environment, accurate aligning of the arrays was very difficult. The aim was to have the arrays parallel, and with the shortest distance being between transmit element one and receive element one, but this could not be ensured in all cases. This has some influence on the small scale statistics, and will be discussed in the following section.

\section{DATA EVALUATION}

For all ten measurements, the measured transfer functions $H(f)$ were inverse Fourier transformed, without any windowing, to the delay domain. This resulted in an impulse response $h(\tau)$ for each of the 49 combinations of transmitter and receiver elements of the ULAs. For each of these, a power delay profile (PDP) was calculated as $|h(\tau)|^{2}$. A cable reflection, occuring at 170 ns delay, and also measured in calibration measurements was manually removed from the measured data. Finally, the 49 PDPs for each of the transmitter-receiver combinations were averaged to obtain 10 averaged power delay profiles (APDPs).

In [6], it has been suggested to adjust the delay axis of the power delay profile so that the (quasi)-LOS component of all measurement points corresponds to the same delay (the required adjustment can be obtained from simple geometrical considerations). Such a correction facilitates the extraction of the $m$-parameters of the first arriving components. However, this procedure was not done in our case, since the inaccuracy of the array alignment rendered this procedure too error-prone.
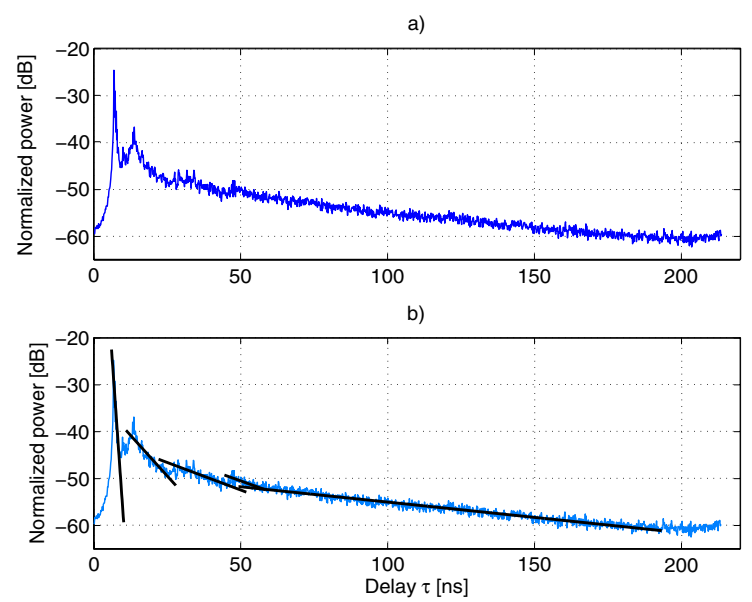

Fig. 2. Averaged power delay profile for a $2 \mathrm{~m}$ LOS measurement, b) also contains regression lines fitted to each cluster whereas a) is without.

\section{RESUlTS}

\section{A. Fit to Saleh-Valenzuela Model}

The measured data are modelled by a Saleh-Valenzuela (S-V) model. In the $\mathrm{S}-\mathrm{V}$ model, the impulse response is given by

$$
h(t)=\sum_{l=0}^{\infty} \sum_{k=0}^{\infty} \beta_{k l} e^{j \theta_{k l}} \delta\left(t-T_{l}-\tau_{k l}\right),
$$

where $\beta_{k l}$ and $\theta_{k l}$ are the gain and phase of the $k$ th ray and $l$ th cluster, respectively, whereas $T_{l}$ is the arrival time of the $l$ th cluster and $\tau_{k l}$ the arrival time of the $k$ th ray measured from the beginning of the $l$ th cluster. The gain is determined by

$$
\overline{\beta_{k l}^{2}} \equiv \overline{\beta^{2}\left(T_{l}, \tau_{k l}\right)}=\overline{\beta^{2}(0,0)} e^{-T_{l} / \Gamma} e^{-\tau_{k l} / \gamma},
$$

where $\Gamma$ and $\gamma$ are the cluster and ray power decay constants, respectively [12]. Our first objective is to determine the ray power decay constants $\gamma$ of our measured data. An important aspect here is that the $\mathrm{S}-\mathrm{V}$ model assumes that the $\gamma$ are the same for all clusters of a certain impulse response. By mere inspection of our APDPs (see Fig. 2a), it can be seen that this is not the case in our measurements [11].

Before determining the decay constants, it is necessary to divide each APDP into clusters. The identification can be performed in several ways: when the clusters are well-separated in the delay domain, it is sufficient to find the maxima of the power delay profile, since these signify the onset of a new cluster. Alternatively, a "best fit" procedure can be used, where the number and start time of clusters is used as parameters that are fitted to the measured power delay profile. This approach was used, e.g., in the parameterization of the IEEE 802.15.3a channel models. However, it can suffer from numerical problems - depending on the choice of the start values of the minimumsearch algorithm, different solutions (that all fit the measurement results) can be obtained. We thus in this paper choose an approach "by visual inspection", as the human eye is good at the detection of patterns and structures even in noisy data. The fact that we have different $\gamma$ for different clusters is of help, since a cluster then 
can be identified as all consecutive components that fit the same regression line on a dB-scale. Hence, by indentifying different slopes, we identify different clusters. This is exemplified in Fig. $2 \mathrm{~b}$, where five different slopes can be observed. The identification process thus immediately gives the ray power decay constant $\gamma_{l}$ of each cluster as $\gamma_{l}=1 / k_{\text {reg, } l}$ where $k_{r e g, l}$ is the negative slope of the regression line belonging to cluster $l$. In all our measurements we have a number of clusters that ranges between 4 and 6 , and $\gamma$ always varies with delay. The $\gamma$ values range from 0.1 and $20.6 \mathrm{~ns}$, with an overall average value of $\bar{\gamma}=5.17 \mathrm{~ns}$, but since there are large differences of the values within a measurement, an average value is not sufficient to describe them. Therefore, the $\gamma$ :s belonging to the same measurement are plotted as a function of delay, where the delay of a certain cluster is defined as the time of the first arriving component of that very cluster, i.e., $T_{l}$ in the $\mathrm{S}-\mathrm{V}$ model. $^{2}$ We thus propose a generalized $\mathrm{S}-\mathrm{V}$ model where $\gamma$ increases linearly with delay (see Fig. 3), i.e.,

$$
\gamma=\gamma(\tau) \propto a \tau
$$

where the average value (including both LOS and NLOS measurements) of the constant $a$ is 0.35 . If only considering the LOS cases, we have $a_{L O S}=0.21$ whereas only NLOS results gives $a_{N L O S}=0.44$. It should be stated that the division into LOS and NLOS may be insufficient, since the NLOS results can look very different in different measurements. The NLOS could be sub-divided into two groups, where the first one contains the measurements of shorter distance, which all have strong first components and look very similar to the LOS cases, and the second one contains the measurements of larger distance, where the cluster powers are not monotonically decreasing. Instead, the latter group shows a behaviour that rather looks as an exponential rise followed by an exponential decay. The "NLOS group 1" results often resemble the LOS results more than the "NLOS group 2" results, but this has not been further used. Only the division into LOS and NLOS has been used since the number of statistical points are not enough to verify a general trend.

In the $\mathrm{S}-\mathrm{V}$ model, the cluster power decay constant $\Gamma$ is determined as the exponential decay of the peak power of the received clusters. By mere inspection of our APDPs (cf. Fig. 2), it can be seen that this will not make a good fit to our data. Instead, we investigate the decay of the normalized total cluster power, i.e., we normalize the total power within each cluster with its ray power decay constant, which gives a better agreement to an exponential decay (see Fig. 4). Hence $\Gamma$ can be determined as the inverse of the negative slope of the regression lines fitting the decay of the total cluster power. This gives an average value of $\bar{\Gamma}=3.62 \mathrm{~ns}$. For LOS we have $\overline{\Gamma_{L O S}}=2.63 \mathrm{~ns}$ and for NLOS, $\overline{\Gamma_{N L O S}}=4.94 \mathrm{~ns}$. However, the modelling of the NLOS data again deserves some extra attention. Since their cluster powers are not monotonically decreasing, the APDPs of the so-called "NLOS group 2" are not suitable for a linear fit as in Fig. 4. Therefore only the results from "NLOS group 1" are used when calculating $\overline{\Gamma_{N L O S}}$.

\footnotetext{
${ }^{2}$ Indeed, there are a few cases where some uncertainty remains regarding exactly when one cluster ends and the next one begins, but this has only a minor effect on our results.
}

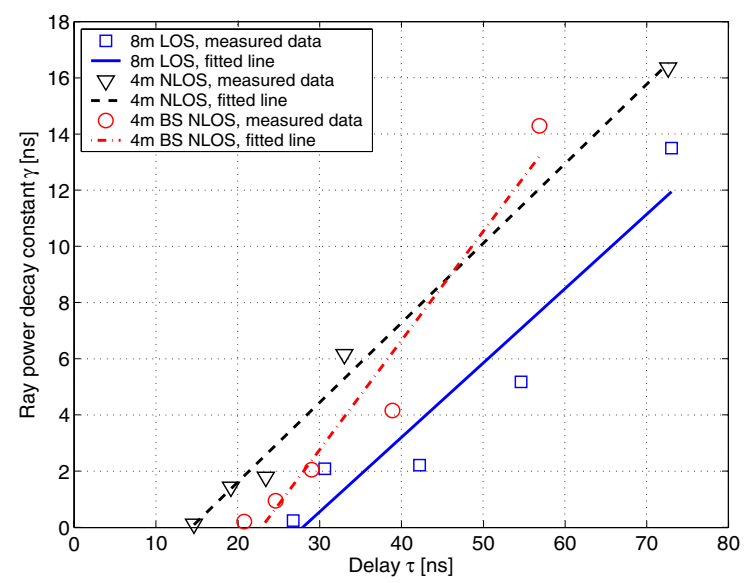

Fig. 3. The ray power decay constant $\gamma$ as a function of delay for three different measurement positions.

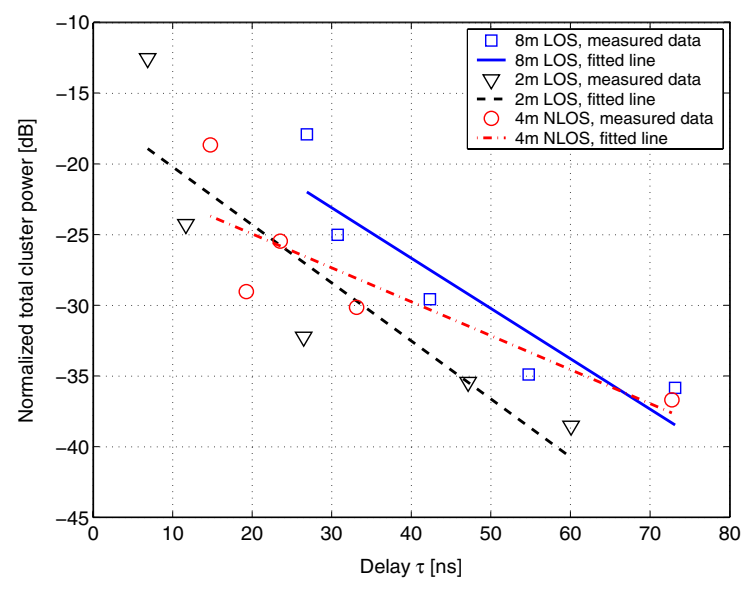

Fig. 4. The normalized total cluster power as a function of delay for three different measurement positions.

The cluster arrival rate $\Lambda$ is obtained by measuring the cluster interarrival times $\Delta T_{l}=T_{l}-T_{l-1}$ for each APDP, with $\Lambda=$ $1 / \overline{\Delta T_{l}}$ where $\overline{\Delta T_{l}}$ is the average value within the APDP. We note that $\Delta T_{l}$ seems to increase with delay in our measurements. However, this is not used any further, since the number of measured $T_{l}$ (which are realizations of a random variable) is not sufficient to allow determination of a general trend for the probability density function. According to the $\mathrm{S}-\mathrm{V}$ model, $\Delta T_{l}$ is described by an exponential distribution and this agrees well with our results (see Fig. 5). In average for all measurements we have $\overline{(1 / \Lambda)}=12.38 \mathrm{~ns}$, with the LOS and NLOS average values being $\overline{(1 / \Lambda)_{L O S}}=14.11 \mathrm{~ns}$ and $\overline{(1 / \Lambda)_{N L O S}}=11.23 \mathrm{~ns}$, respectively.

The ray arrival rate $\lambda$ is not determined since, despite a delay resoultion of $0.13 \mathrm{~ns}$, it was not possible to resolve the inter-path arrival times by inverse Fourier transform of the measured data.

The Rice factor $K_{r}$ for each cluster is determined as

$$
K_{r}=\frac{P_{k \mid \max }}{\left(\sum_{k} P_{k}\right)-P_{k \mid \max }},
$$




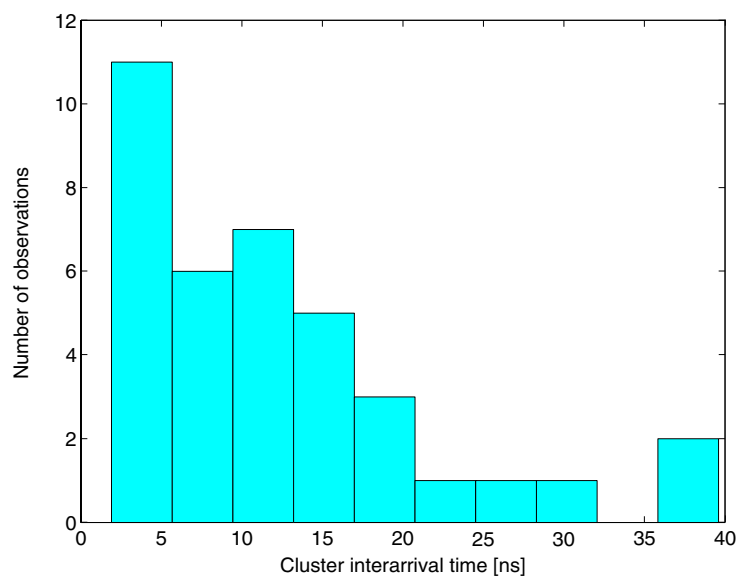

Fig. 5. A histogram of the cluster interarrival times for all measurement points.

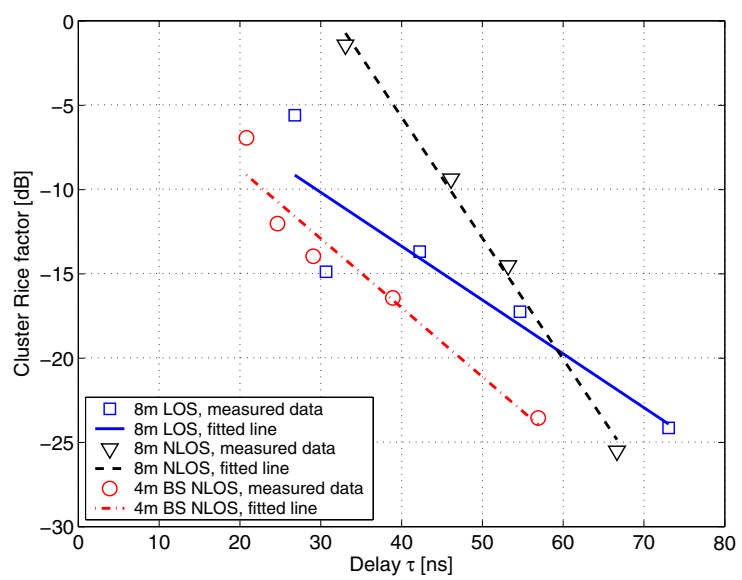

Fig. 6. The cluster Rice factor as a function of delay for three different measurements.

where $P_{k \mid \max }$ is the strongest component of the cluster, and the summation is made over all $k: \mathrm{s}$ within the cluster. This gives values in the range of $-25.6 \mathrm{~dB}$ to $0.5 \mathrm{~dB}$ for all measurements. The average value of the Rice factor is $\overline{K_{r}}=-14.2 \mathrm{~dB}$ (overall, as well as for the LOS and NLOS cases, respectively), but as in the case of the ray power decay constants it is necessary to also describe the dependence over delay. If $K_{r}$ is plotted as a function of the delay (i.e., the arrival time of the first component of each cluster), it can be seen that it seems to follow a linear (in $\mathrm{dB}$ ) decrease with increasing delay (see Fig. 6).

\section{B. Small-Scale Statistics}

In [6], it is stated that for an indoor channel the energy that falls within a certain delay bin is $m$-Nakagami distributed. In order to analyse if this is the case for our measurements, the 49 amplitude values $\left|h_{i}\right|(i=1,2, \ldots 49)$ are fitted to the $m$ Nakagami distribution using the $m$-estimates given by the inverse normalised variance (INV) estimator [13],

$$
\widehat{m}_{I N V}=\frac{\mu_{2}^{2}}{\mu_{4}-\mu_{2}^{2}},
$$

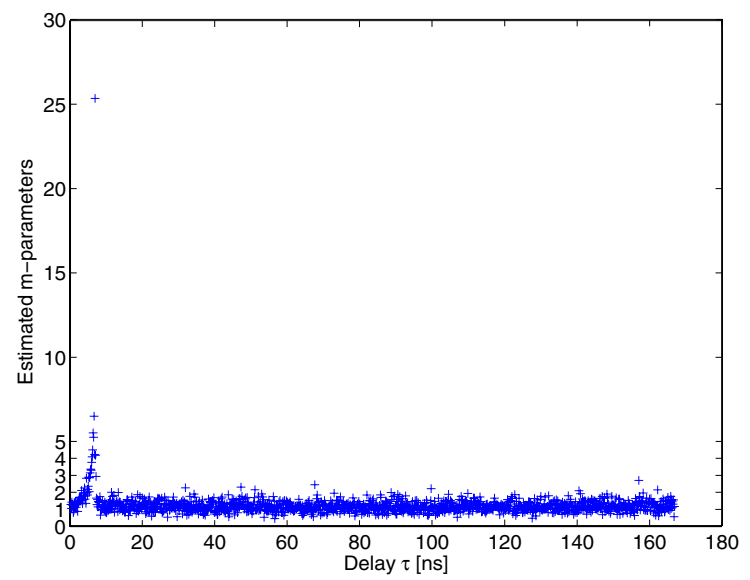

Fig. 7. The $m$-parameter estimates for each delay bin of a $2 \mathrm{~m}$ LOS measurement.

where $\mu_{k}=N^{-1} \sum_{i=1}^{N}\left|h_{i}\right|^{k}$. For most of the measurements, the best fit distribution seems to have an $m$-value of 1 , which corresponds to a Rayleigh distribution. The only exception is for the LOS component and some nanoseconds before (see Fig. 7). This is clearly different from the office environment in [6], where the $m$-parameter is found to be decreasing with the delay. In our measurements a Rayleigh distribution is applicable at all delays (except for the LOS component). This can be explained by the much higher density of scatterers in the industrial environment. We also note that it is markedly different from the 802.15.3a channel model (designed for office environments) where a lognormal distribution was assumed for all multipath components.

\section{Rake Receiving}

For the design of Rake receiver systems, it is important to know the number of multipath components (MPCs) to be collected in order to capture a certain amount of the energy. Our analysis shows the difficulty of designing a Rake receiver for an industrial environment. For distances of $8 \mathrm{~m}$ in a NLOS scenario, collecting the 100 strongest MPCs would still only capture a little more than $10 \%$ of the total energy (see Fig. 8). This demonstrates the challenges of designing UWB systems in industrial environments.

\section{Summary AND CONCLUSIONS}

We have investigated ultrawideband propagation channels in an industrial environment, and established a statistical model that describes the behavior of the channel. We found that the power delay profile can be well described by a Saleh-Valenzuela model (with model parameters given in Table I), which is also used in the IEEE 802.15.3a channel models. There are several noteworthy points:

- In contrast to the classical S-V model, the ray power decay constants depend on the maximum excess delay. This dependence is well described by a linear relationship. The decay constants vary between 0.1 and $20 \mathrm{~ns}$.

- The total cluster power is an exponential function of the excess delay.

- The number of clusters varies between 4 and 6 . 


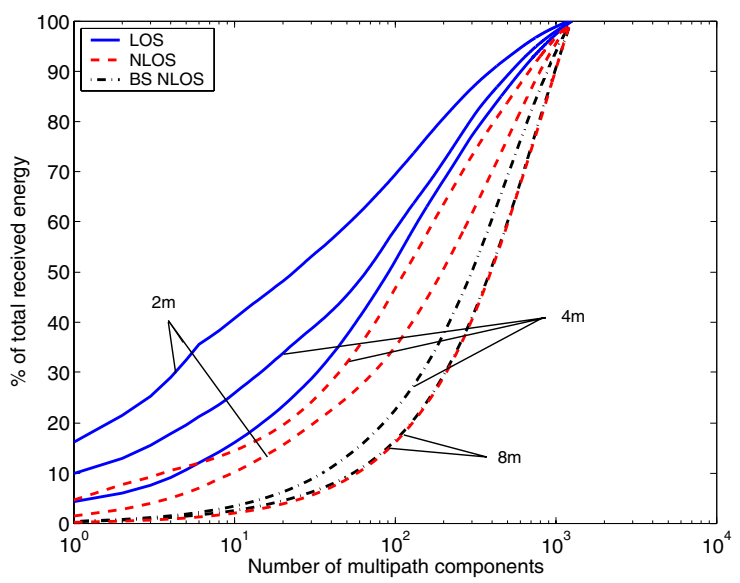

Fig. 8. The received energy for a certain number of multipath components.

TABLE I

FITTED SALEH-VALENZUELA MODEL PARAMETERS

\begin{tabular}{|c|c|c|c|c|}
\hline \multirow{2}{*}{} & \multicolumn{4}{|c|}{ Saleh-Valenzuela parameters } \\
\cline { 2 - 5 } & $1 / \Lambda$ & $\Gamma$ & $\gamma$ & $\gamma(\tau)$ \\
\hline LOS & $14.11 \mathrm{~ns}$ & $2.63 \mathrm{~ns}$ & $4.58 \mathrm{~ns}$ & $\propto 0.21 \tau$ \\
NLOS & $11.23 \mathrm{~ns}$ & $4.94 \mathrm{~ns}$ & $5.58 \mathrm{~ns}$ & $\propto 0.44 \tau$ \\
\hline
\end{tabular}

- The small-scale fading is well described by a Rayleigh distribution, except for the first components in each cluster, which can show a strong specular contribution.

- The inter-path arrival times were so small that they were not resolvable even with a delay resolution of $0.13 \mathrm{~ns}$.

Additionally, we found that the number of MPCs that is required for capturing $50 \%$ of the energy of the impulse response can be very high, up to 400 . This serves as motivation to investigate suboptimum receiver structures that do not require one correlator per MPC, e.g., transmitted-reference schemes, [14], [15], as well as noncoherent schemes. We also find that a considerable percentage of the received energy lies outside a $60 \mathrm{~ns}$ wide window; this is important in the context of a current IEEE 802.15.3a standardization proposal, which uses OFDM with a 60 ns guard interval.

Our results emphasize the crucial importance of realistic channel models for system design. They will be used as an input to the IEEE 802.15.4a channel modelling group, which (among other issues) develops a channel model for industrial environments.

\section{ACKNOWLEDGEMENTS}

We thank DSM Resins Scandinavia for their permission to perform the measurements in their factory hall. Especially, we would like to thank Mr. Bengt-Åke Ling, Mr. Gert Wranning, and Mr. Alf Jönsson for their help and cooperation. Part of this work was financially supported by the Swedish Research Council and an INGVAR grant of the Swedish Strategic Research Foundation.

\section{REFERENCES}

[1] R. A. Scholtz, "Multiple access with time-hopping impulse modulation," in Proc. IEEE Military Comm. Conf., vol. 2, pp. 447-450, Oct 1993.

[2] M. Z. Win and R. A. Scholtz, "Ultra-wide bandwidth time-hopping spreadspectrum impulse radio for wireless multiple -access communications," IEEE Trans. Comm., vol. 48, pp. 679-691, Apr 2000.
[3] C. J. LeMartret and G. B. Giannakis, "All-digital PAM impulse radio for multiple-access through frequency-selective multipath," in Proc. IEEE Global Telecomm. Conf., pp. 77-81, 2000.

[4] Federal Communications Commission, "First report and order 02-48," tech. rep., 2002.

[5] M. Z. Win and R. A. Scholtz, "Impulse radio: How it works," IEEE Comm. Lett., vol. 2, pp. 36-38, Feb 1998.

[6] D. Cassioli, M. Z. Win, and A. F. Molisch, "The ultra-wide bandwidth indoor channel: From statistical models to simulations," IEEE J. Selected Areas Comm., vol. 20, pp. 1247-1257, Aug. 2002.

[7] S. Ghassemzadeh, R. Jana, C. Rice, W. Turin, and V. Tarokh, "Measurement and modeling of an ultra-wide bandwidth indoor channel," IEEE Trans. Comm., 2004. in press.

[8] A. F. Molisch, J. R. Foerster, and M. Pendergrass, "Channel models for ultrawideband personal area networks," IEEE Personal Comm. Magazine, 2003 , in press.

[9] T. S. Rappaport, S. Y. Seidel, and K. Takamizawa, "Statistical channel impulse response models for factory and open plan building radio communication system design," IEEE Trans. on Comm., vol. 39, pp. 794-807, May 1991.

[10] R. C. Qiu, "A generalized time domain multipath channel and its application in ultra-wideband (UWB) wireless optimal receiver design: Wave-based system ananlysis," Trans. Wireless Comm., 2003, in press.

[11] J. Karedal, S. Wyne, P. Almers, F. Tufvesson, and A. F. Molisch, "UWB channel measurements in an industrial environment," Globecom 2004, in press.

[12] A. A. M. Saleh and R. A. Valenzuela, "A statistical model for indoor multipath propagation," IEEE J.Selected Areas Comm., vol. 5, pp. 128-137, Feb. 1987.

[13] A. Abdi and M. Kaveh, "Performance comparison of three different estimators for the Nakagami m parameter using monte carlo simulation," IEEE Comm. Lett., vol. 4, pp. 119-121, Apr. 2000.

[14] J. D. Choi and W. E. Stark, "Performance of ultra-wideband communications with suboptimal receivers in multipath channels," IEEE J. Selected Areas Comm., vol. 20, pp. 1754-1766, Dec. 2002.

[15] F. Tufvesson and A. F. Molisch, "Ultra-wideband communication using

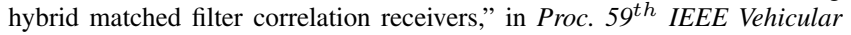
Technology Conference, 2004. 